Recepción: 27 / 12 / 2017

Aceptación: 16 / 02 / 2018

Publicación: 08 / 05 / 2018
Ciencias de la economía y empresariales Artículo de Revisión

\title{
Las redes sociales en el emprendimiento de una marca comercial
}

Social networks in the undertaking of a commercial brand

Redes sociais no empreendimento de uma marca comercial

\author{
Hernán G. Carrión I \\ hgcarrion1206@yahoo.es \\ Silvana E. Jiménez-Pereira II \\ sejimenezp@gmail.com \\ Ylse Duran ${ }^{\text {III }}$ \\ ylsemoreladg@gmail.com
}

Correspondencia: ylsemoreladg@gmail.com

\footnotetext{
${ }^{\text {I }}$ Magister en Educación y Desarrollo Social, Ingeniero Comercial, Licenciado en Administración de Empresas, Universidad San Gregorio de Portoviejo, Portoviejo, Ecuador.

${ }^{\text {II }}$ Magister en Administración de Empresas, Ingeniero en Administración de Empresas, Docente de la Universidad Nacional de Loja, Loja Ecuador.

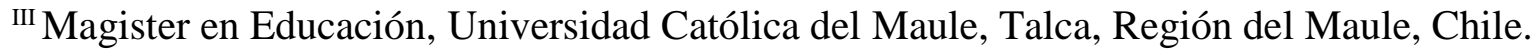




\title{
Resumen
}

El trabajo realizó una revisión teórica, relacionado con las variables redes sociales y emprendimiento. Se utilizó una metodología descriptiva documental relacionadas con los tipos y uso de las redes sociales afines con el emprendimiento. Es de destacar que el uso de las redes, representa un medio publicitario accesible para cualquier persona y por lo tanto la inversión que el emprendedor debe realizar para introducir su marca es prácticamente nula comparado con la inversión en medios tradicionales como los medios impresos, tv o la radio y puede ser asumido sin costo alguno desde la primera etapa de introducción en el mercado. Entre sus conclusiones, se determinó que las redes constituyen un medio de uso de los emprendedores para adquirir diversidad de información en beneficio de sus marcas. Las redes sociales dan oportunidades de innovación en los emprendedores, facilitándoles aperturas de nuevos productos y de las necesidades comerciales de la sociedad.

Palabras clave: Redes sociales; emprendimiento; red social de emprendimiento; marca.

\begin{abstract}
The work carried out a theoretical review, related to the variables social networks and entrepreneurship. A documentary descriptive methodology related to the types and use of social networks related to entrepreneurship was used. It is noteworthy that the use of networks represents an advertising medium accessible to any person and therefore the investment that the entrepreneur must make to introduce his brand is practically nil compared to the investment in traditional media such as print, TV or radio and can be assumed at no cost from the first stage of introduction in the market. Among its conclusions, it was determined that networks constitute a means of use by entrepreneurs to acquire diversity of information for the benefit of their brands. Social networks provide opportunities for innovation in entrepreneurs, facilitating openings of new products and the commercial needs of society.
\end{abstract}

Keywords: Social networks; entrepreneurship; entrepreneurial social network; brand.

\section{Resumo}

O trabalho realizou uma revisão teórica, relacionada às variáveis redes sociais e empreendedorismo. Foi utilizada uma metodologia descritiva documental relacionada aos tipos e uso de redes sociais relacionadas ao empreendedorismo. Vale ressaltar que o uso de redes, 
representa um meio de publicidade acessível para qualquer pessoa e, portanto, o investimento que o empreendedor deve tomar para introduzir sua marca é insignificante em comparação com o investimento em meios tradicionais, como mídia impressa, TV ou rádio e pode ser assumido sem custo a partir da primeira fase de introdução no mercado. Entre suas conclusões, determinou-se que as redes constituem um meio de uso por empreendedores para adquirir diversidade de informações em benefício de suas marcas. As redes sociais proporcionam oportunidades de inovação nos empreendedores, facilitando a abertura de novos produtos e as necessidades comerciais da sociedade.

Palavras chave: Redes sociais; empreendedorismo; rede social empreendedora; marca.

\section{Introducción}

Actualmente, el crecimiento acelerado de las redes sociales ha permitido la incorporación de un sin número de sitios sociales en internet y la frecuencia de uso es permanente, ante la diversidad de programas que ofrece, que van desde la búsqueda de un trabajo hasta el marketing de diversas marcas. De allí, que para el mundo del emprendimiento comercial resulta ser una herramienta de mucha utilidad.

Cabe destacar, al emprendimiento como una forma de pensar y de actuar, que tiene su origen en las personas que se esfuerzan permanentemente por convertir sus pensamientos y palabras en acciones concretas que generen valor para sí mismas y para la comunidad que les rodea. Resulta pertinente, para aquellos que hacen de las dificultades y adversidades un elemento catalizador necesario para inspirarse y lograr un cambio positivo en sus vidas.

Asimismo, apuntan Hoang y Antoncic (2003), que las redes sociales emergen como una importante área dentro del campo del emprendimiento. Pues, la masificación de la Internet y su evolución continúan influenciando día a día a más personas, que no sólo aprovechan las bondades de redes sociales como Facebook y Twitter y otras para comunicarse, sino que también las utilizan para explorar las opciones que existen en el mercado y sobre esa base, un emprendedor, indica Baum et al (2000), inmerso en el proceso de empezar algo nuevo, requiere asegurar que su iniciativa sea reconocida como legítima socialmente y así obtener el soporte social requerido; entre más grande sea la innovación, mayor será la necesidad de reconocimiento y aceptación 
social; por lo tanto, el emprendedor usará su posición en la red con el propósito de derribar las barreras que le impiden ganar la legitimidad necesaria para obtener dicha aceptación social.

\section{Redes sociales}

Las redes sociales digitales (RSD), redes sociales virtuales o social network sites son un servicio basado en una plataforma web que permite a las personas construir un perfil público o semipúblico dentro de un sistema acotado, articular una lista de otros usuarios con quien se quiere compartir una conexión, y ver y cruzar su lista de contactos y las hechas por otros dentro del sistema (Boyd \& Ellison, 2007).

La plataforma tecnológica, según Leimeister et al. (2006), permite y apoya la interacción de la comunidad y ayuda a construir confianza y un sentimiento común entre los miembros. Una RSD puede ser vista como una estructura de intercambio social con su propia estructura de gobierno y patrones de interacción, en la cual los recursos fluyen entre unidades independientes o individuos.

\section{Las Redes sociales más utilizadas}

Según datos recopilados de Statistic Brain y expandedramblings.com, las redes sociales más usadas en el 2017 son:

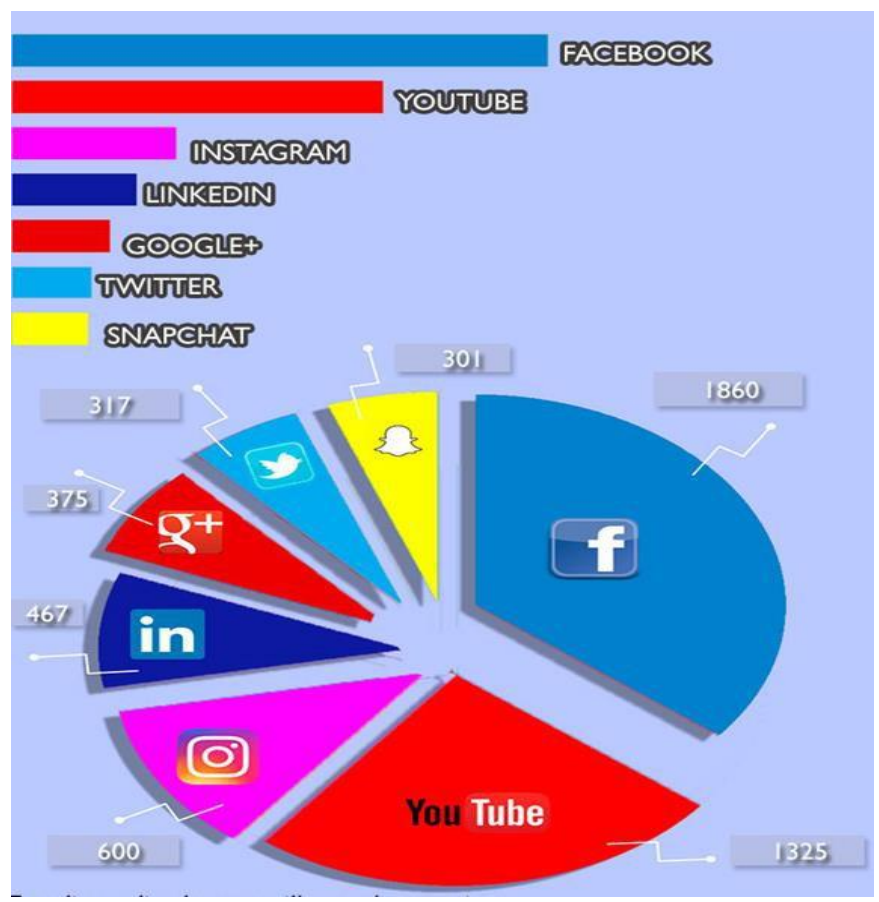

Figura1. Redes sociales de mayor uso. ( Statistic Brain y expandedramblings.com) 
Las redes sociales propician la interacción de miles de personas en tiempo real, con base en un sistema global de relaciones entre individuos basados en la estructura social de Georg Simmel (1950). Si tenemos en cuenta que toda actividad humana genera consecuencias jurídicas, podemos afirmar que las redes sociales no son otra cosa que máquinas sociales diseñadas para fabricar situaciones, relaciones y conflictos con multitud de efectos jurídicos.

\section{Direccionalidad de las redes sociales}

Se puede afirmar que no existen criterios de unificación en cuanto a los tipos de redes sociales que actualmente se manejan. Sin embargo, regularmente se consideran dos direccionalidades que las agrupan, una de tipo horizontal y la otra vertical.

La direccionalidad Horizontal refiere a todo tipo de usuarios y sin una temática definida. Se basan en una estructura de celdillas permitiendo la entrada y participación libre y genérica sin un fin definido, distinto del de generar masa. Los ejemplos más representativos del sector son Facebook, Orkut, Identi.ca, Twitter, Google+, Hi5, ello o Bebo. Las redes sociales horizontales se dirigen a todo tipo de usuarios y no tienen una temática definida ni un fin concreto.

La direccionalidad vertical, están concebidas sobre la base de un eje temático agregador. Su objetivo es el de congregar en torno a una temática definida a un colectivo concreto. En función de su especialización, pueden clasificarse a su vez en:

- Redes sociales Verticales Profesionales: Están dirigidas a generar relaciones profesionales entre los usuarios. Los ejemplos más representativos son Viadeo, Xing y Linked In.

- Redes sociales Verticales de Ocio: Su objetivo es congregar a colectivos que desarrollan actividades de ocio, deporte, usuarios de videojuegos, fans, etc. Los ejemplos más representativos son Wipley, Minube, Dogster, Last.FM y Moterus.

- Redes sociales Verticales Mixtas: Ofrecen a usuarios y empresas un entorno específico para desarrollar actividades tanto profesionales como personales en torno a sus perfiles: Yuglo, Unience, PideCita, 11870” (Burgueno, 2009).

Cabe destacar que, las redes sociales verticales, por basarse en un tema concreto, permiten congregar un gran número de usuarios en torno a una temática concreta. 


\section{Tipos de redes sociales}

Cuando nos referimos a redes sociales, el concepto abarca más que Facebook o Twitter, simplemente porque los sitios que permiten hacer networking son esencialmente sociales. Existen muchas clases de sitios y entre ellos tenemos:

\section{- Foros:}

Probablemente el primer formato de red social que hubo en Internet, permiten la interacción social entre personas que comparten intereses comunes, como puede ser fotografía, programación o música.

Los usuarios mantienen conversaciones sobre temas particulares y construyen relaciones en base a sus opiniones en el foro. Son muy buenas fuentes de conocimiento sobre los temas a los que se dedican y es una plataforma ideal para compartir lo que sabes

\section{- Blogs:}

Originalmente llamados web-logs, los blogs son una plataforma para que las personas expresen sus ideas y sus vidas, los hay como diarios y otros dedicados a nichos y temas particulares.

\section{- Micro-blogging:}

Aunque parezcan similares a los blogs, en este tipo de sitio lo que se busca es hacer una versión pequeña y resumida de lo que está sucediendo ahora mismo, puede tratarse de pensamientos o noticias, cualquier tipo de información que el usuario quiera compartir. El microblog más conocido es sin dudas Twitter.

\section{- Para compartir fotos:}

Si bien se puede compartir fotos en todas las redes sociales, existen plataformas especiales para fotografías, ya que su interface y funcionalidades son más útiles para quienes desean compartir cantidades considerables de imágenes, como que cada foto tiene un enlace propio y se puede etiquetar con palabras claves para que la gente pueda comentar. Los más populares son Flickr y Picasa. 
- Para compartir videos:

YouTube es el sitio para compartir vídeos que todos conocen, los vídeos se comparten en línea, palabras claves y etiquetas se agregan para facilitar las búsquedas, además que se permite la interacción de usuarios mediante comentarios y la personalización del perfil.

Otros sitios similares sonVimeo y MetaCafe.

- Profesionales:

Hacer contactos o networking siempre ha sido una necesidad en el mundo de los negocios, ya que conectándose con otros profesionales una persona puede hacer nuevas conexiones a través de ellos. Lo que obviamente conlleva a nuevas oportunidades, a afirmarse en un campo y a no perder contacto con ex compañeros de trabajo y/o jefes. La más conocida es LinkedIn, pero existen muchas otras redes sociales para empresas y negocios.

\section{- Para Socializar:}

Las redes sociales propiamente dichas permiten mantener contacto con otras personas y que incluso hacer nuevas amistades, ya que justamente en estos sitios se trata de ser sociales, el líder en esta categoría es Facebook.

La vulnerabilidad de las redes sociales ha permitido, entre sus múltiples usos, emprender actividades empresariales, como iniciativa de sus usuarios en pro del desarrollo de un proyecto de negocios o una idea en particular que genere ingresos, crear microempresa, entre otras.

\section{Emprendimiento desde las redes sociales}

El mundo digital, constituye una gran oportunidad para el mundo del emprendimiento, pues se constituye en un canal de comunicación.

Afirma Patiño (2012), tienen que tener una red social porque son muy económicas, permiten segmentar audiencias, conocer públicos, analizar información del consumidor y obtener métricas relevantes para el negocio. Eso sí, deben tener claro que el alcance de la presencia siempre deberá estar medida por el modelo de negocio que se tenga" afirma Patiño, quien complementa que en este momento se evidencia el ingreso de empresas de diferentes sectores al mundo digital, pero se hace más latente en vestuario, diseño y alimentos. 


\section{Enfoques de redes emprendimiento desde las sociales}

Smith y Lohrke (2007), mencionan tres enfoques desde los cuales se puede estudiar la incidencia de las redes en el proceso de emprendimiento, entre estos, el estructuralista, relacional o cognitivo, coinciden en que las redes tienen una influencia importante sobre el proceso de emprendimiento. Los emprendedores con ausencia de esas relaciones, generalmente enfrentan riesgos subyacentes a la dificultad de ganar confianza y apoyo de los proveedores de recursos clave, quienes perciben el riesgo de selección adversa procedente de la asimetría de información y la falta de legitimidad.

Aldrich y Ruef (2006), reconocen la importancia de las redes preexistentes de los emprendedores, ellas son el medio primario de acceso a recursos necesarios durante la etapa previa a la fundación. Una parte importante de estas redes preexistentes está constituida por las relaciones de carácter familiar. La importancia de la red familiar es fundamental; Anderson, Jack y Drakopoulou (2005), en su estudio del caso escocés, encontraron que cerca de la cuarta parte de los lazos clave de las redes de emprendimiento corresponden a vínculos familiares.

\section{Tipos de redes sociales de emprendimiento}

Una red social es una estructura social que se puede representar a través de uno o varios grafos, al respecto Barabási, (2002), indica que los grafos o redes tienen propiedades ocultas bajo su estructura, que limitan o multiplican la capacidad para hacer cosas con ellas y ayudan a reducir la incertidumbre asociada con la actividad emprendedora. Por su parte, Hung (2006) resalta la existencia de dos tipos de redes de mucho interés para el emprendimiento: las redes interpersonales y las redes interorganizacionales.

- Las redes interpersonales: se refieren a redes personales del emprendedor, mientras que las interorganizacionales se refieren a redes extendidas. Una red interpersonal denota cómo a nivel personal los emprendedores están impregnados en varios sistemas sociales. Los emprendedores usarán sus redes interpersonales para relacionarse con otros individuos o grupos, buscando acceso a recursos y fuentes de información relevante, en su propósito de convertir una idea en una entidad de negocio. Las relaciones interpersonales del emprendedor están constituidas básicamente por los sistemas sociales en los cuales está inmerso, tales 
como su familia, amigos, colegas, sus contactos de negocios y las afiliaciones a clubes o asociaciones profesionales.

- Las redes interorganizacionales: muestran cómo un new entrepreneurial venture ya establecido, se relaciona con otras organizaciones, después de que ha sido creado, formado y desarrollado por su fundador. Hansen (1995), sugiere que estructuralmente las redes interorganizacionales corresponden a redes de colaboración de tipo informal, y que son consideradas como una forma alternativa de coordinación interorganizacional frente a los mercados y jerarquías. De otro lado, una red interorganizacional es un mecanismo estratégico para mejorar la ventaja competitiva de una empresa a través de la minimización de los costos de transacción, mientras se mantiene la flexibilidad que permite acelerar la innovación tecnológica.

Cuando las relaciones son de largo plazo, las transacciones de carácter reiterativo y existe algún tipo de especificidad de activos (Williamson, 1991), se genera interdependencia, la cual puede ser también una explicación de la formación de redes de cooperación interorganizacional, debido a que en este caso cumplen con tres funciones específicas: regular las transacciones interfirmas, desarrollar una visión de futuro compartida y proveer una infraestructura de coordinación (Hung, 2006).

\section{Metodología}

De acuerdo con el objeto de estudio, el cual lo constituyeron las diferentes teorías relacionadas con el uso de las redes sociales y el emprendimiento, esta investigación se puede enmarcar dentro de una investigación descriptiva - documental, esta última a través de la recopilación y análisis de bibliografía e información de ambas variables. Finalmente, considerando un enfoque hermenéutico y bajo el uso de la técnica de triangulación de las teorías revisadas, fue posible extraer argumentos teóricos que se presentan de manera conclusiva. También, fue posible realizar un análisis descriptivo de la información aportada por 58 emprendedores de diferentes marcas comerciales. 


\section{Análisis de los resultados}

Posterior a la información aportada por 58 informantes emprendedores de diferentes marcas comerciales, fue posible procesar la información y presentar las siguientes tablas con sus respectivos gráficos.

Tabla 1. Análisis descriptivo de la variable redes sociales

\begin{tabular}{lllll}
\hline \multicolumn{1}{c}{ ETAPAS } & Fr & \% & FRA & \%A \\
\hline Redes Sociales Verticales & 14 & 15,9 & 14 & 15,9 \\
\hline Redes Sociales Horizontales & 33 & 37,5 & 47 & 53,4 \\
\hline Redes Sociales Mixtas & 41 & 46,6 & 88 & 100 \\
\hline Total & $\mathbf{8 8}$ & $\mathbf{1 0 0}$ & $\mathbf{8 8}$ & $\mathbf{1 0 0}$ \\
\hline
\end{tabular}

Fuente: Entrevista a emprendedores.

En la tabla 1 se presenta la distribución frecuencial y porcentual de las variables redes sociales como producto del análisis conjunto de las dimensiones redes sociales horizontales, verticales y mixtas, en lo que respecta al uso de las redes sociales mixtas, en la misma se observa que el $46,6 \%$ de los encuestados coincidieron en que utilizan de igual forma tantas las redes sociales horizontales como las verticales para dar a conocer sus marcas comerciales. Sin embargo, el $37,5 \%$ consideró de mayor uso las redes sociales de tipo verticales y el 15,9\% indicó a las redes sociales horizontales.

Asimismo, al triangular las teorías se pudo constatar entre los resultados que la direccionalidad Horizontal pudo haber sido la más favorecida, dado que refiere a todo tipo de usuarios y sin una temática definida, a través de medios masificados como el Facebook, Orkut, Identi.ca, Twitter, Google+, Hi5, ello o Bebo. 
Tabla 2. Análisis descriptivo de la variable emprendimiento

\begin{tabular}{|c|c|c|c|c|}
\hline ETAPAS & $\mathbf{F R}$ & $\%$ & FRA & $\% A$ \\
\hline Redes interpersonales & 17 & 19,3 & 17 & 19,3 \\
\hline Redes interorganizacional & 31 & 35,2 & 48 & 54,5 \\
\hline Redes Mixtas & 40 & 45,5 & 88 & 100 \\
\hline Total & 88 & 100 & 88 & 100 \\
\hline
\end{tabular}

Fuente: Entrevista a emprendedores.

En la tabla 2 se presenta la distribución frecuencial y porcentual de la variable emprendimiento, como producto del análisis conjunto de las dimensiones redes interpersonales, redes interorganizacionales y redes mixtas, en lo que respecta al uso de las redes mixtas el 45,5\% consideró la de mayor uso. Seguido del 35,2\% que indicó las redes interorganizacionales dada su informalidad, y como una forma alternativa de coordinación interorganizacional frente a otros mercados y jerarquías y el 19,3\% señalo a las redes interpersonales para relacionarse con otros individuos o grupos, buscando acceso a recursos y fuentes de información relevante, en su propósito de convertir una idea en una entidad de negocio.

Al triangular la información, fue posible determinar que el uso combinado de las redes interpersonales y las interorganizacionales tienen prevalencias, en tanto que al complementarse suman más inserción a la comunicación social, si consideramos que las primeras cubren el total del entorno social del emprendedor de la marca y las segundas está a la orden de sus competencias y comercios afines.

\section{Conclusiones}

- $\quad$ Existe un alto número de redes que permiten estar en contacto con los amigos, saber lo que están haciendo en cada momento, compartir con ellos fotos y vídeos, entre otras cosas, considerado por los emprendedores de suma importancia para sus marcas.

- Las redes sociales representan un medio publicitario de muy bajo costo para cualquier persona y por lo tanto no tiene derogación económica para que un emprendedor pueda 
introducir su marca en el mundo empresarial, si lo comparamos con la inversión en medios tradicionales como los medios impresos, TV o la radio.

- $\quad$ Fue posible constatar, que las redes sociales se constituyen como un medio de uso de los emprendedores para adquirir diversidad de información en beneficio de sus marcas.

- $\quad$ Las redes sociales dan oportunidades de innovación a los emprendedores, facilitándoles aperturas de nuevos productos y de las necesidades comerciales de la sociedad.

\section{Referencias bibliográficas}

Baum, J. (2000). The effect of so- cial networks on resource access and bu- siness start-ups. European Planning Stu- dies, 10(8), 1039-1046.

Hoang. H. \& Antoncic B. (2003). The evolution of firm networks: From emergence to early growth of the firm. Strategic Management Journal, 22, 275-286.

Hung, H. (2006). Network-based research in intrepreneurship. A critical re- view. Journal of Business Venturing, 18, 165-187.

Simmel, G. (1950). Fundamental Problems of Sociology, pp-3-84 in Kurt H. Wolff (ed.), The Sociology of Georg Simmel. New York: Free Press.

Smith, D. \& Lohrke, F. (2007). Entrepreneurial network development: Trusting in the pro- cess. Journal of Business Research, en prensa.

Williamson, O. (1991). Comparative economic or- ganization: The analysis of discrete structural alternatives. Administrative Science Quarterly, 36, 269-296. 